\title{
Fibromodulin Ablation Exacerbates the Severity of Acute DSS Colitis
}

Mariana Halasi, $\mathrm{PhD}^{1}$; Mor Grinstein, $\mathrm{PhD}^{2}$; Avner Adini, $\mathrm{PhD}^{3}$; Irit Adini, $\mathrm{PhD}^{1, *}$

1. Harvard Medical School, Department of Surgery, Center for Engineering in Medicine \& Surgery, Massachusetts General Hospital, 51 Bloosm Street, Boston, MA 02114 USA

2. Harvard Medical School, Center for Regenerative Medicine, Massachusetts General Hospital, Boston, MA 02114. Email: mgrinstein@mgh.harvard.edu

3. Harvard Medical School, Department of Medicine, Boston Children's Hospital, Karp Research Building, 11th Floor 300 Longwood Ave, Boston, MA 02115, Email:

avner.adini@,childrens.harvard.edu

* Correspondence to: Irit Adini, Department of Surgery, Harvard Medical School, Center for Engineering in Medicine \& Surgery, Massachusetts General Hospital, 51 Bloosm Street, Boston, MA 02114 USA. Phone: 617.371.4874; Fax: 617.573.9471; Email: iadini@mgh.harvard.edu 
bioRxiv preprint doi: https://doi.org/10.1101/2022.01.12.475640; this version posted January 12, 2022. The copyright holder for this preprint (which was not certified by peer review) is the author/funder. All rights reserved. No reuse allowed without permission. 


\begin{abstract}
Epidemiological studies have linked pigment production to protection against certain human diseases. In contrast to African Americans, European descendants are more likely to suffer from angiogenesis-dependent diseases, and inflammatory diseases such as wet age-related macular degeneration (ARMD) and ulcerative colitis (UC), respectively.

Here, we found that albino mice producing high levels of fibromodulin (FMOD) developed less severe acute colitis than mice lacking FMOD as assessed by the clinical symptoms and the histopathological changes. In a dextran sodium sulfate (DSS)-induced acute colitis mouse model, depletion of FMOD affected the expression and localization of tight junction proteins contributing to destruction of the epithelial barrier. Furthermore, this study demonstrates the development of a stronger inflammatory response after DSS treatment in the absence of FMOD. FMOD depletion led to an increase in activated T cells, plasmacytoid dendritic cells (pDCs), and type I IFN production.

These findings strongly suggest that FMOD may serve as a potential biomarker in determining disease severity of UC in the population of light-skinned individuals with European descent.
\end{abstract}

Keywords: FMOD, colitis, tight junction, inflammation, dendritic cells, T cells 


\section{INTRODUCTION}

Ulcerative colitis (UC) is an inflammatory bowel disease (IBD), affecting primarily the mucosal surface of the large intestine (colon and rectum). UC is characterized by the destruction of the intestinal barrier, the disappearance of intestinal crypts, and the infiltration of inflammatory cells ${ }^{1,2}$. Epidemiological studies indicate that the prevalence of UC continues to increase worldwide, particularly in developed countries. Individuals with North American and Northern European ancestry have the highest incidence and prevalence rates with 20/100,000 and 156 - 291/100,000 cases per year, respectively. Multiple factors contribute to UC pathogenesis, including environmental factors, genetic predisposition, changes in intestinal microbiota, and immune system dysfunction ${ }^{3,4}$.

UC progression is mainly affected by the dysregulation of tight and adherens junction proteins that form the apical junction complex and maintain the epithelial barrier. Damage to the junction proteins results in dysfunctional mucosal barrier and increases intestinal permeability ${ }^{5}$. The molecular composition of the tight junctions (TJs) includes transmembrane proteins (claudin, occludin), cytoplasmic proteins (zonula occludens-1; ZO-1), and cytoskeletal components ${ }^{6}$. These TJs prevent the internalization of intestinal pathogens. Interestingly, Dendritic cells (DC), the most effective antigen presenting cells that play a key role in maintaining immunological homeostasis by orchestrating innate and adaptive immune responses, also express TJ proteins. DCs are found at the interfaces with the external environment, like in the intestinal mucosa, where they act as sentinels for external pathogens ${ }^{7-9}$.

Fibromodulin (FMOD), is a small leucine-rich proteoglycan that plays a role in extracellular matrix (ECM) composition. FMOD was shown to activate the classical complement cascade by direct binding to $\mathrm{C} 1 \mathrm{q}$ and by the deposition of $\mathrm{C} 3 \mathrm{~b}, \mathrm{C} 4 \mathrm{~b}$ and $\mathrm{C} 9$. FMOD can also silence the complement cascade by binding to factor $\mathrm{H}^{10,11}$. Our previous work had shown that FMOD up-regulates TGF- $\beta 1$ secretion ${ }^{12-14}$. TGF- $\beta 1$ appears to enhance intestinal epithelial barrier function by inducing the production of TJ proteins, such as claudin-1, occludin and ZO-1

15. Furthermore, it is well known that TGF- $\beta 1$ is key to maintain DCs in an immature state ${ }^{16}$. During activation and migration of immature DCs to the inflammatory sites, DCs interact directly with ECM microenvironment proteins, such as FMOD, leading to DC differentiation and maturation ${ }^{17,18}$. 
To advance our understanding of UC pathogenesis, we investigated the role of FMOD in the DSS-induced murine model of acute colitis and found that FMOD depletion results in severe pathological colitis. 


\section{METHODS}

\section{Mice}

Mutant B6(Cg)-Tyr $r^{c-2 J / J}(\mathrm{FMOD}+/+)$ 8-10-week-old male mice were purchased from the Jackson Laboratory. The mutant B6(Cg)-Tyr $r^{c-2 J} / \mathrm{J}$ transgenic FMOD knockout (FMOD-/-) animals were established previously as described ${ }^{12}$. The knockout animals were bred and maintained in pathogen-free conditions in the animal facility of Massachusetts General Hospital, Boston, MA. All animals were housed on $12 \mathrm{hr}$ light-dark cycles under controlled temperatures, and they had free access to standard diet and water. All animal experiments were approved by and were conducted in accordance with the guidelines of the Institutional Animal Care and Use Committee at Massachusetts General Hospital.

\section{Induction of experimental DSS colitis}

Acute colitis was induced in age-, and gender- matched, parental FMOD+/+ and FMOD-/- mice by the administration of 3\% DSS (molecular weight 36,000-50,000; MP Biomedicals) ad libitum in autoclaved drinking water for 7 days. Body weight, stool consistency and bleeding were recorded daily to monitor colitis development. On day 7 after the start of DSS treatment, the animals were sacrificed, the gross pictures of the colon from cecum to rectum were taken, colon length was measured, and the colon tissue samples were processed for further downstream analysis.

\section{Human samples}

Colon biopsy sections of healthy individuals and patients diagnosed with ulcerative colitis or

Crohn's disease were purchased from the Biorepository and Tissue Bank of the University of Massachusetts, Center for Clinical and Translational Science (UMCCTS).

\section{Scoring of bleeding}

Bleeding score criteria follows as: $0=$ no blood; $1=$ small blood spot around anus; $2=$ visible rectal bleeding; $3=$ dried, chunks of blood on fur around anus. 


\section{Scoring of Disease Activity Index (DAI)}

DAI was calculated by the addition of the average weight loss score, the average stool consistency score and the average bleeding score and then divided by 3 . Weight loss score criteria follows as: $0=$ no weight loss; $1=1-5 \%$ weight loss; $2=6-10 \%$ weight loss; $3=11-20 \%$ weight loss. Stool consistency score follows as: $0=$ normal; $1=$ loose stool; $2=$ elongated loose stool; $3=$ diarrhea.

\section{Scoring of H\&E sections}

On the day of termination, the colon was removed, flushed with ice cold phosphate buffered saline (PBS) to remove feces and blood, cut longitudinally and prepared as a swiss roll for fixation in 4\% paraformaldehyde (PF). Paraffin embedded sections were stained with Hematoxylin and Eosin (H\&E) and histological scoring was performed in a blinded fashion independently by two pathologists. The histological sections were examined for the integrity of tissue architecture (0-4), ulceration (0-4), and submucosal edema (0-4).

\section{Total RNA extraction and quantitative real-time PCR (qRT-PCR)}

Colon tissue samples were processed by the IBI Isolate reagent (IBI Scientific) for total RNA extraction. The High-Capacity cDNA Reverse Transcription Kit (Applied Biosystems) was used to synthesize complementary DNA (cDNA). Quantitative real-time PCR reactions were run on the LightCycler 480 instrument (Roche) using the PrimeTime Gene Expression Master Mix (IDT DNA) and the following mouse primers: Claudin1-S, 5 -ACA GCA TGG TAT GGA AAC AGA-3`, Claudin1-AS, 5 `-AGG AGC AGG AAA GTA GGA CA-3; IFN $\alpha / \beta-S, 5^{`}$-TTC CTC AGC CAA GTT GCT-3`, IFN $\alpha / \beta$-AS, 5 `-GTG CAG TGT CCT AGT CCA G-3`; Ocludin-S, 5 `CAC TAT GAA ACA GAC TAC ACG ACA-3`, Ocludin-AS, 5 '-GTT GAT CTG AAG TGA TAG GTG GA-3`; TJP1-S, 5`-AAT GAA TAA TAT CAG CAC CAT GCC-3`, TJP1-AS, 5`GCC ACT ACA GTA TGA CCA TCC-3`; 18S-S, 5 -GAG ACT CTG GCA TGC TAA CTA G3`, 18S-AS, 5 `-GGA CAT CTA AGG GCA TCA CAG-3`; GAPDH-S, 5 `-AAT GGT GAA GGT CGG TGT G-3`, GAPDH-AS, 5 `GTG GAG TCA TAC TGG AAC ATG TAG-3`.

\section{Cell isolation and Flow cytometry}

Mesenteric lymph nodes ( $\mathrm{mLN}$ ) of DSS treated animals were isolated, passed through a $100-\mu \mathrm{m}$ 
cell strainer and red blood cells were lysed with the RBC lysis buffer (Biolegend). To differentiate between live and dead cells, cells were stained for $15 \mathrm{~min}$ at room temperature with the Zombie UV Fixable Viability Kit (Biolegend) as per company recommendation. Cell surface staining was performed for $30 \mathrm{~min}$ at $4^{\circ} \mathrm{C}$ with fluorochrome-conjugated monoclonal antibodies to mouse antigens purchased from Biolegend: CD45 (30-F11), CD11c (N418), PDCA-1 (927), CD3 (17A2), CD4 (GK1.5), CD25 (PC61). Following staining cells were washed and fixed in 4\% PF.

For intracellular staining, cells first were stained for cell surface markers, washed, then fixed, permeabilized and stained for intracellular transcription factor FoxP3 (MF-14; Biolegend) for 30 min at RT. Samples were analyzed by the FACSAria cell sorter (BD Biosciences), and the acquired data were analyzed using FlowJo software (version 10.6.1).

\section{Microscopy and Immunofluorescence staining}

Brightfield images of H\&E-stained sections were captured by Nikon Eclipse Ti2 microscope. For immunofluorescence staining, paraffin embedded colon sections were rehydrated by incubating in a series of xylene, ethanol $(100 \%, 95 \%, 70 \%$ and 50\%) and phosphate-buffered saline (PBS) solutions. After blocking, primary antibodies of CD45 (Biolegend), claudin-1 (BiCell Scientific), FMOD (Abcam) occludin (BiCell Scientific) or ZO-1 (BiCell Scientific) were applied for overnight incubation. As for secondary antibodies, Alexa Fluor 594 (Invitrogen) and Alexa Fluor 647 (Invitrogen) were utilized. To visualize nuclei, slides were mounted with VECTASHIELD Antifade mounting medium with DAPI (Vector Laboratories). Fluorescence imaging was performed with Nikon Eclipse Ti2 microscope or Leica SP8 X confocal microscope. Acquired images were analyzed using the NIS Elements software (Nikon) or the LASX life science software (Leica). Mean Fluorescence Intensity (MFI) was calculated by ImageJ 8.

\section{Statistical analysis}

Statistical analysis was performed with Graphpad as described in the figure legends. $P$ values of $<0.05$ were considered to be statistically significant. 


\section{RESULTS}

\section{FMOD expression is affected by ulcerative colitis in human subjects and mice}

The expression of FMOD was assessed by immunostaining in colon biopsy samples from healthy control individuals and patients who had been diagnosed with UC or Crohn's disease (CD). As shown in Fig.1A left panel, in healthy individuals, FMOD is mainly detected in the brush border on the luminal cell surface of the colon. In contrast, a significant increase in FMOD expression was observed in the crypts of UC patient colon biopsies (Fig.1A right panel). Since DSS-induced colitis is a well-established and commonly used animal model 19,20 which mirrors key aspects of human IBD, especially UC, FMOD level and localization was 
evaluated in animals with DSS-induced colitis. FMOD expression demonstrated the same distribution as observed in human samples (Fig.1B).

A
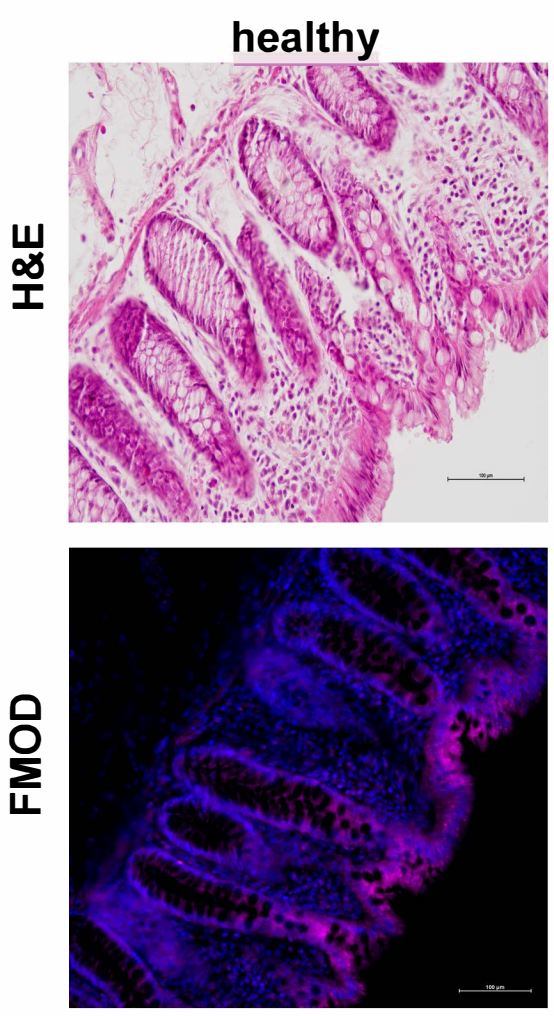

B

$\mathbf{B}$

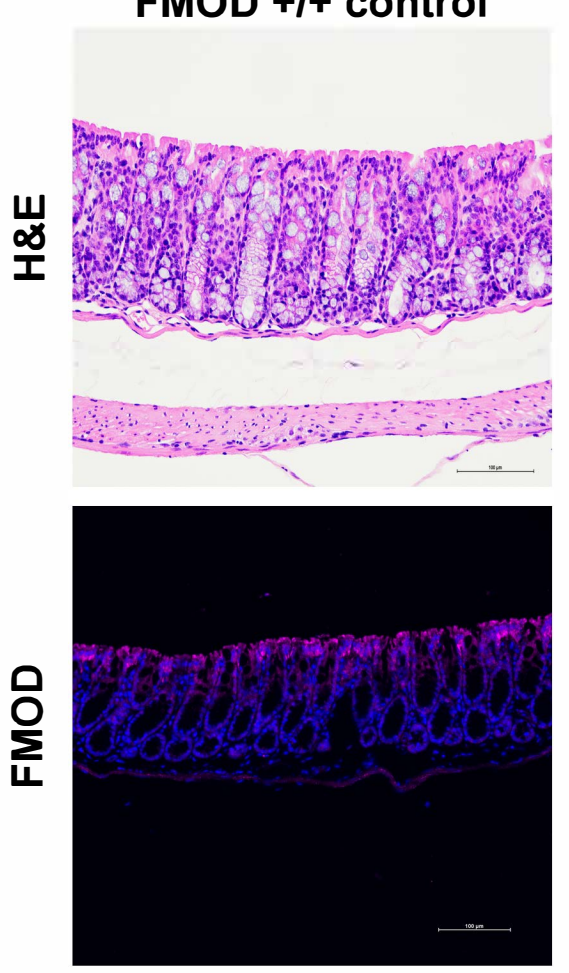

\section{ulcerative colitis}
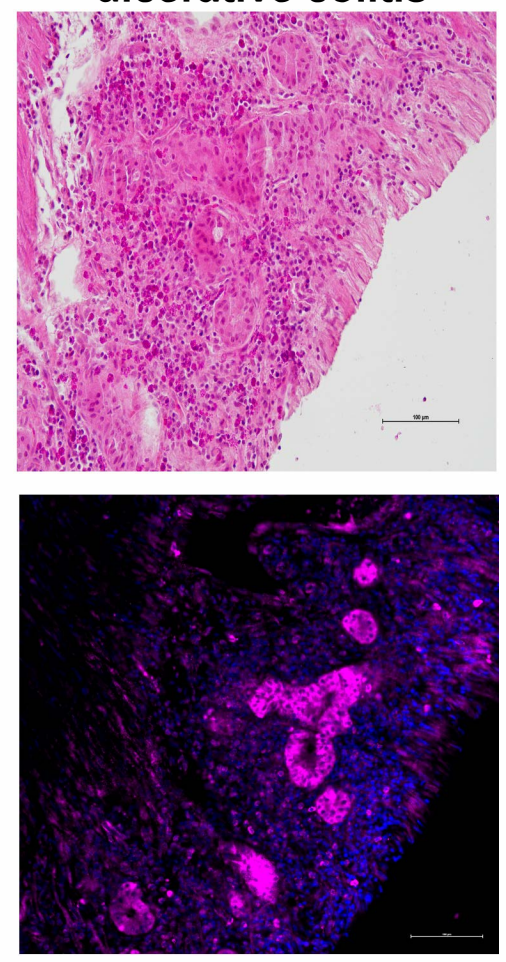

FMOD +/+ DSS
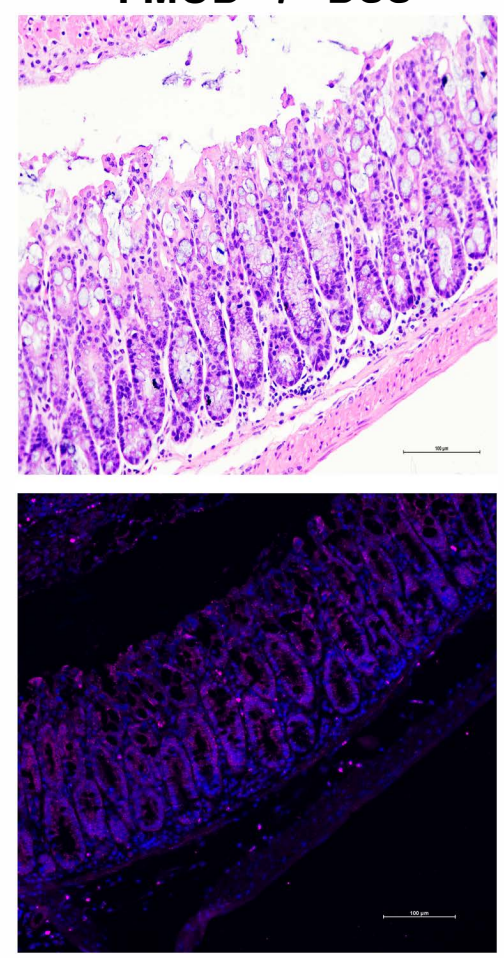
Figure 1. FMOD is upregulated and re-localized in colitis. (A) Colon biopsy samples from healthy individuals and patients diagnosed with UC were H\&E stained or were subjected to immunostaining for FMOD. Representative images were captured at x20 magnification. (B) FMOD $+/+$ mice were administered 3\% DSS in their drinking water for 7 days. Representative samples were H\&E stained or immunostained for FMOD. Images were obtained at x20 magnification.

\section{Depletion of FMOD aggravates the DSS-induced clinical symptoms of colitis}

To determine the role of FMOD in the development of acute colitis, age-matched, transgenic FMOD knockout (FMOD-/-) male mice along with the FMOD+/+ animals were exposed to 3\% DSS for 7 days as described in materials and methods. The clinical signs of DSSinduced acute colitis, including change in body weight, bleeding, and fecal consistency, were recorded daily to monitor the onset and progression of the disease. Both FMOD+/+ and FMOD/- DSS-treated experimental animals showed similar disease metrics of acute colitis manifested as body weight loss (Fig. 2A) and shortening of the colon (Fig. 2B, C). However, DSS treated FMOD-/- mice began losing weight 3 days after DSS administration, while the FMOD+/+ animals started showing weight loss 5 days later. During the remainder of the DSS treatment, more substantial weight loss was observed among all treated mice, reaching similar levels of $8.37 \%$ and $10 \%$ loss of the original weight in FMOD+/+ and FMOD-/- animals, respectively (Fig. 2A).

One of the key hallmarks of chemically induced colitis is the shortening of the colon. Like weight loss, colon shortening was similar between the treated groups on the day of necroscopy, 19.71\% in FMOD+/+ and 20.29\% in FMOD-/-, but significantly shorter compared to their respective untreated controls (Fig. 2B, C). Furthermore, both DSS treated groups were visually checked for signs of rectal bleeding. Similar to the weight loss results, the onset of bleeding occurred earlier in the DSS treated FMOD-/- animals, on day 3 compared to day 4 in the FMOD+/+ mice (Fig. 2D). But rectal bleeding remained more prominent in the FMOD-/animals over the period of DSS treatment. The disease activity index (DAI) score including the average of weight loss score, stool consistency score and bleeding score as shown in Fig 2E indicated that FMOD-/- animals showed signs of colitis earlier and developed more severe disease than FMOD+/+ mice. 
Together, these results demonstrate that in FMOD knockout animals, the severity and onset of the clinical symptoms of colitis such as weight loss, rectal bleeding, and diarrhea were exacerbated. 


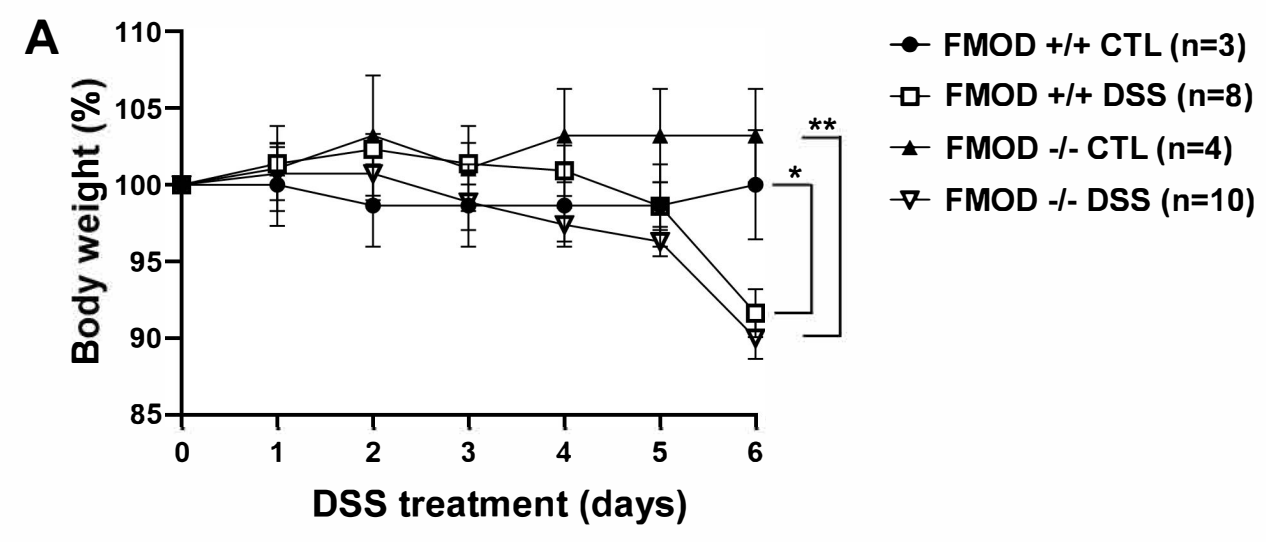

$\mathbf{B}$

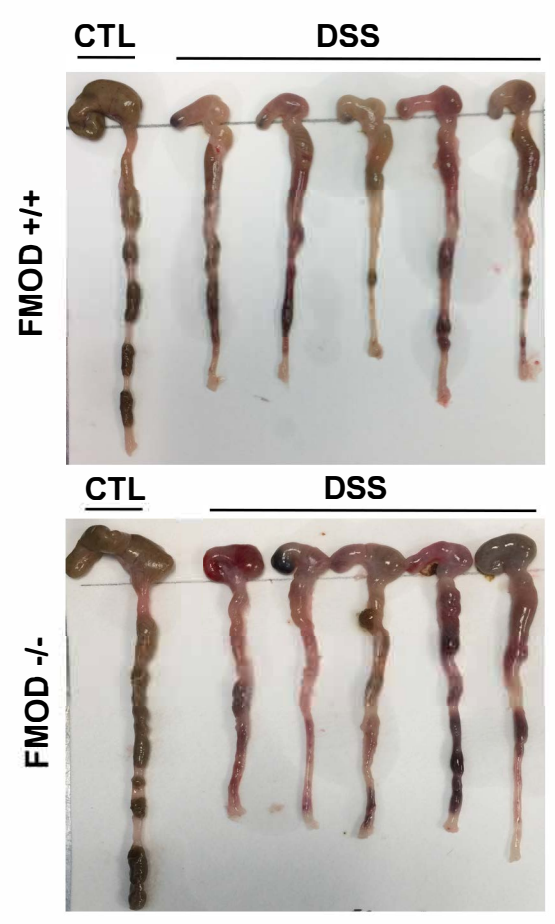

C
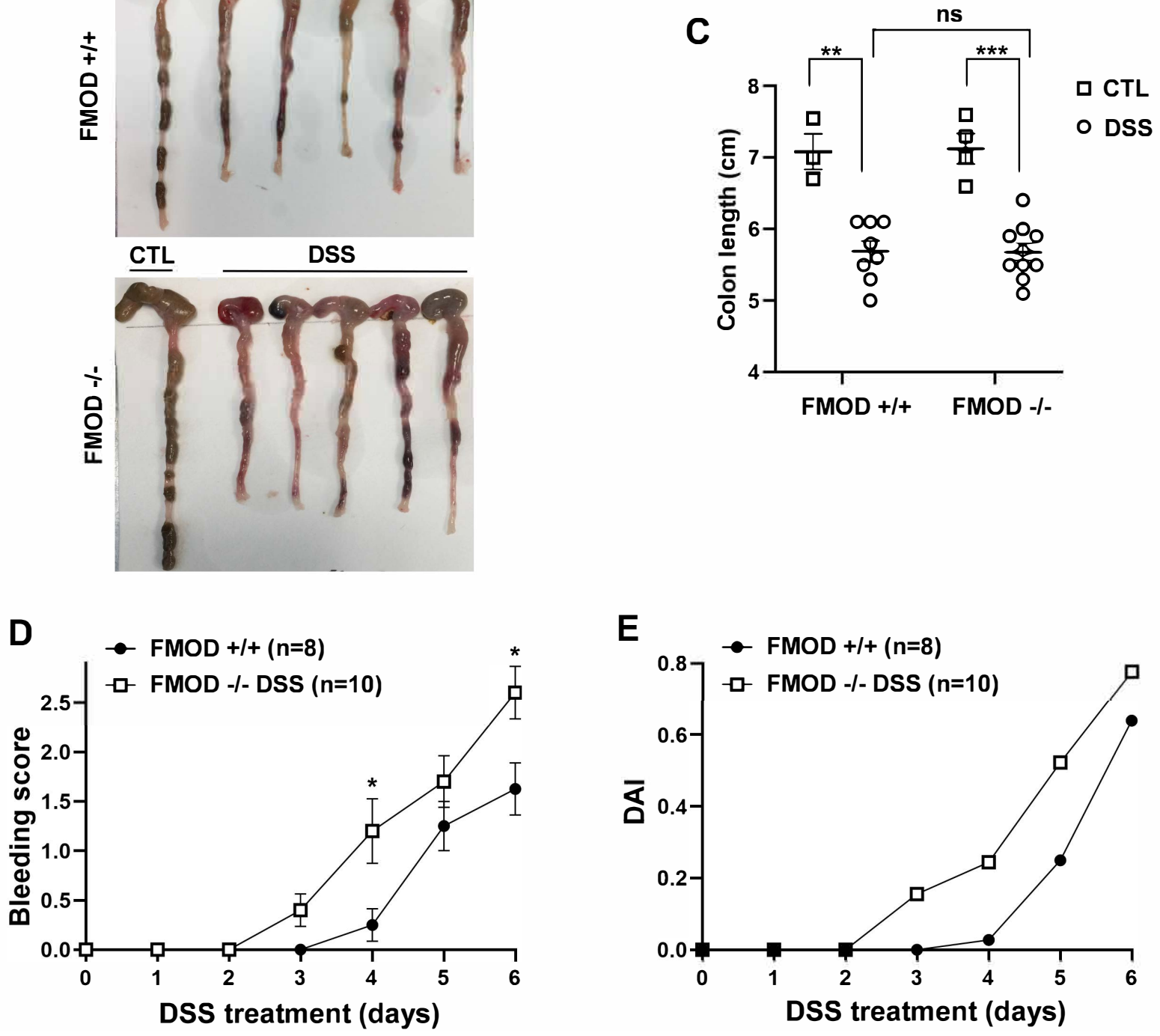

Figure 2. Ablation of FMOD facilitates DSS-related clinical signs of colitis. (A-C) 
FMOD $+/+(n=8)$ and transgenic FMOD-/- $(n=10)$ mice were exposed to 3\% DSS in their drinking water for 7 days, and disease progression was monitored every day. Body weight was recorded daily (A), gross pictures of the colons were taken (B), and colon length was measured and plotted (C) on the day of sacrifice. (D) Rectal bleeding was recorded daily and scored according to the bleeding score criteria detailed in the materials and methods after the visual examination of the anus area of the treated animals. (E) Disease Activity Index (DAI) scores consisted of the sum of the average weight loss score, the average stool consistency score and the average bleeding score and divided by 3 . Data are the mean $\pm \mathrm{SEM}$ from two independent experiments. Statistical analysis was performed by unpaired, two-tailed $t$ test with Welch's correction for (D) and one-way ANOVA with Tukey's multiple comparisons test for (A and C): $* P<0.05, * * P<0.001, * * * P<0.0001$.

\section{FMOD-ablated mice exhibit severe pathological changes of the colon after DSS treatment}

Beside the phenotypical signs of colitis, histopathological changes in the colon were examined to further evaluate the possible role of FMOD in DSS-induced acute colitis. For histological hematoxylin \& eosin (H\&E) staining, the flushed and longitudinally cut colons were prepared as Swiss rolls because this allowed assessing colonic pathology to a fuller extent when compared to the limited scope of transverse or longitudinal sections. Histological observations were carried out to assess the structural integrity of the intestinal epithelium (Fig. 3A). Fully intact epithelium with well-defined crypts, no edema, no leukocyte infiltration in the mucosa and sub-mucosa, no ulceration or erosions were observed on the colon sections of the control animals in either group (Fig. 3A). In contrast, the colon sections of DSS treated animals showed a gut barrier compromised to different degrees between the FMOD+/+ and FMOD-/- groups. Typical histological changes, including the presence of mononuclear inflammatory cells, disappearance of goblet cells, degradation of the epithelium, ulceration, edema, leukocyte infiltration in the mucosa and sub-mucosa, were noted at different levels among the DSS-treated groups (Fig. 3A).

In addition, the colon samples were scored blindly for the following criteria: destruction of architecture (0-4), ulceration (0-4), and edema (0-4) (Fig. 3B). The detailed quantitative histological analysis revealed more advanced disease in the DSS treated FMOD-/- mice with overall histological score average 2.9 compared to the FMOD+/+ with overall histological score average 2 (Fig. 3B). 
Taken together, these results suggest that although FMOD+/+ and FMOD -/- mice show similar physical characteristics of acute colitis, they demonstrate marked differences in colonic histopathology after DSS treatment, in which FMOD-/- animals showed higher susceptibility to DSS and developed more severe colitis. The histological analysis underscores a role for FMOD to moderate the damage inflicted by DSS on the epithelium, since knockout of FMOD resulted in substantial further damage. 
bioRxiv preprint doi: https://doi.org/10.1101/2022.01.12.475640; this version posted January 12, 2022. The copyright holder for this preprint (which was not certified by peer review) is the author/funder. All rights reserved. No reuse allowed without permission.
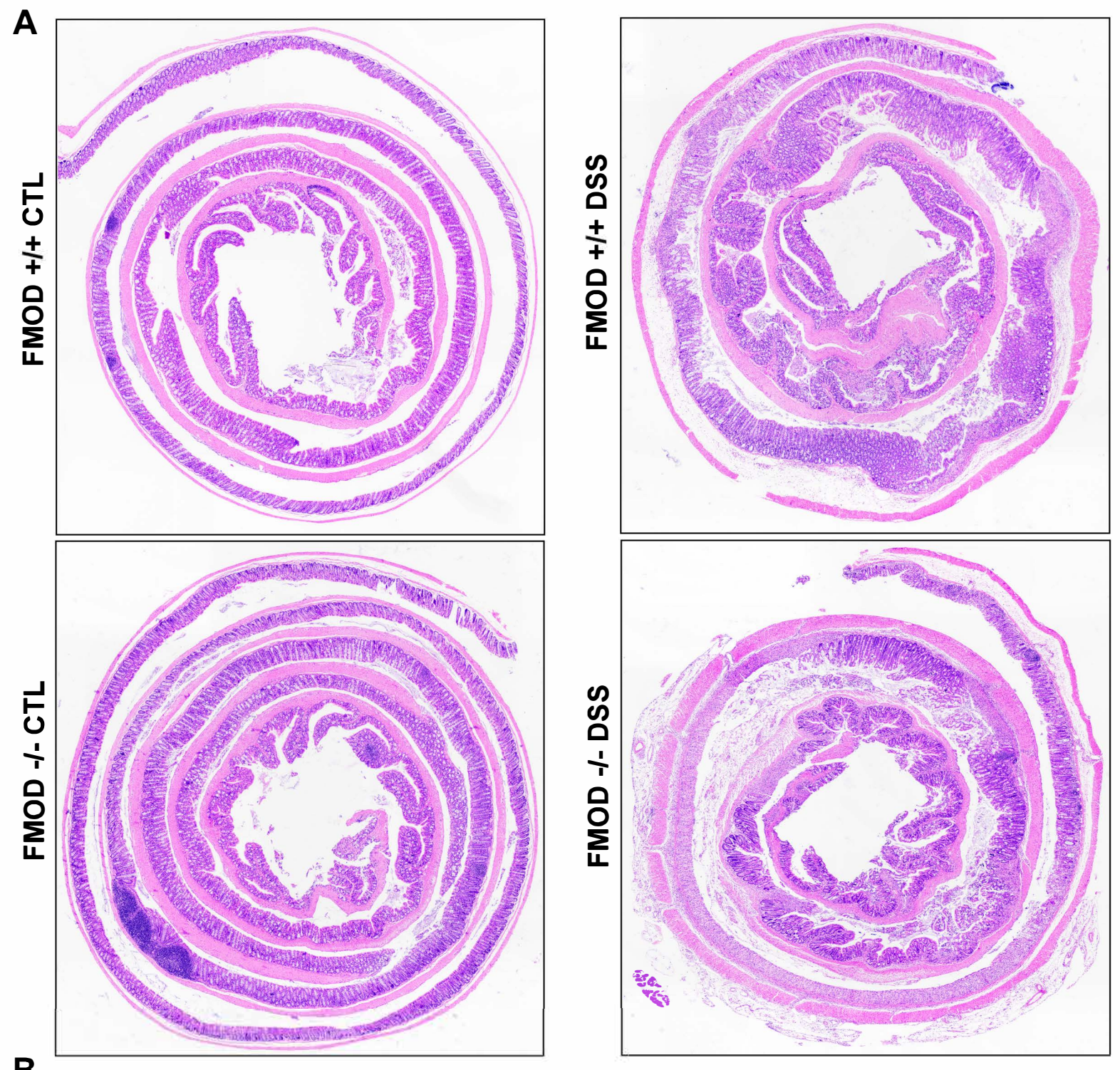

B

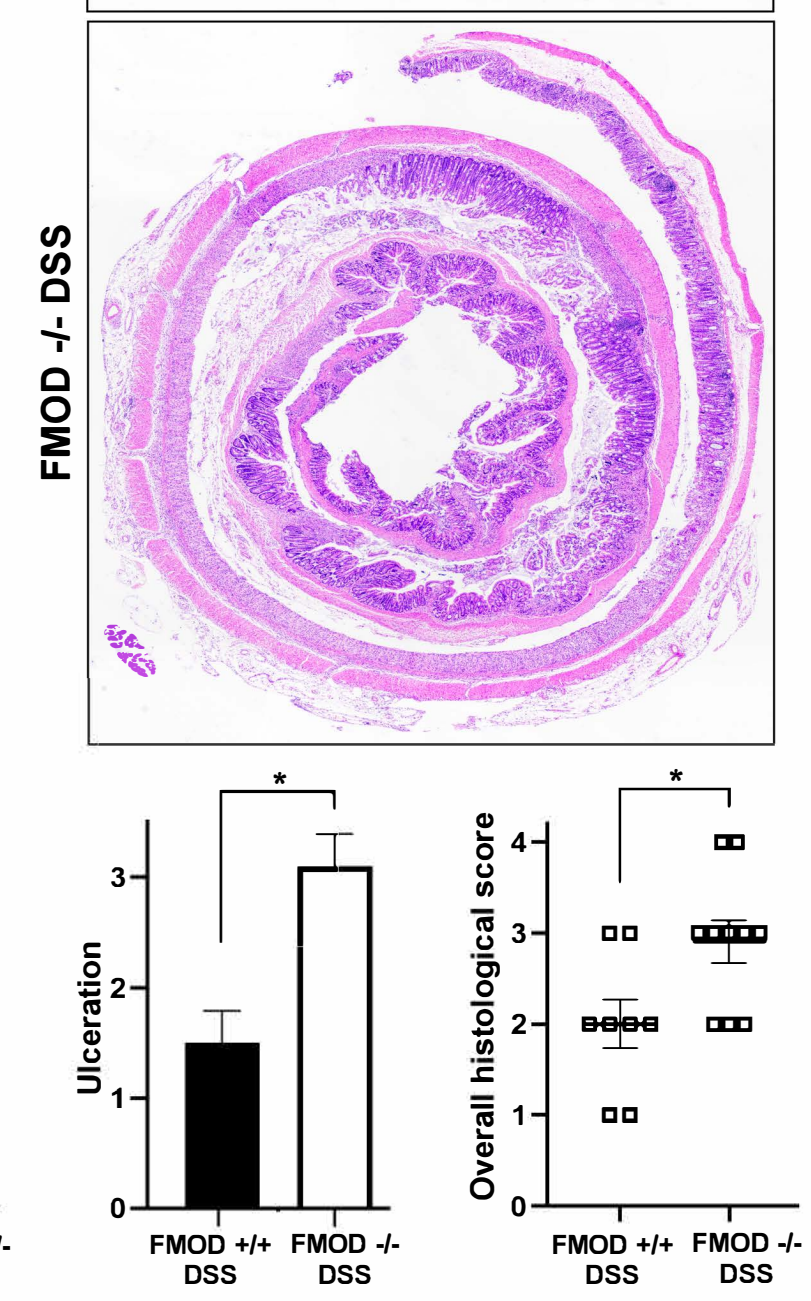




\section{Figure 3. FMOD depletion facilitates DSS-induced pathological changes in the colon. (A)} FMOD+/+ $(\mathrm{n}=8)$ and FMOD-/- $(\mathrm{n}=10)$ mice were subjected to 3\% DSS in their drinking water

for 7 days. Paraffin embedded sections of the colon Swiss rolls were H\&E stained. Images were obtained with $\mathrm{x} 4$ magnification. (B) Histological scores for the destruction of architecture (0-4), edema (0-4), ulceration (0-4), and overall score (0-3) of the full-length colon given in a blinded fashion are plotted. Data are represented as the mean \pm SEM of 3 control and 8 treated of FMOD $+/+$, and 4 control and 10 treated of FMOD-/- animals. $* P<0.05$ by unpaired, two-tailed $t$ test with Welch's correction.

\section{FMOD ablation facilitates epithelial barrier erosion}

By its nature, DSS has the capacity to compromise the structural integrity of the epithelial cell barrier, possibly due to its interference with the TJ proteins ${ }^{21}$. To evaluate the effects of FMOD on the composition and expression of the TJ proteins in DSS-induced colitis, colonic samples of untreated and DSS treated groups were analyzed by immunofluorescence and qRTPCR.

Our analysis focused on intracellular protein ZO-1, and transmembrane proteins occludin and claudin-1 because of their well-established involvement in the impairment of the intestinal barrier function in IBD disease models ${ }^{22,23}$. ZO-1 protein expression was reduced, while claudin-1 expression was substantially upregulated (Fig. 4A), and occludin expression did not show a significant change (data not shown) in the DSS treated colon sections of FMOD-/- mice when compared to treated FMOD+/+ mice. Furthermore, quantification of fluorescence intensity by ImagJ 8 showed a 40\% decrease in ZO1 protein expression and a 1.6-fold increase of claudin1 expression in DSS treated FMOD-/- mice as compared to treated FMOD+/+ mice (Fig.4B). Due to changes in their protein's expression levels, we asked transcriptional regulation or through post-transcriptional events therefore we also validated theses findings at the mRNA level. The results showed that the mRNA level of ZO-1 was significantly reduced, claudin-1 mRNA was substantially upregulated, while occludin mRNA expression did not show any statistically significant change in the DSS treated FMOD-/- mice (Fig. 4C).

Poritz et. al. ${ }^{21}$ found that occludin levels decrease and claudin-1 levels increase in the colon of human subjects with $\mathrm{UC}$, resulting in a higher claudin-1: occludin $(\mathrm{C}: \mathrm{O})$ ratio when compared to the colon of healthy individuals. Using the $\mathrm{C}: \mathrm{O}$ ratio calculated from the respective 
bioRxiv preprint doi: https://doi.org/10.1101/2022.01.12.475640; this version posted January 12, 2022. The copyright holder for this preprint (which was not certified by peer review) is the author/funder. All rights reserved. No reuse allowed without permission.

mRNA expression levels, we observed a 3-fold increase in disease severity in the absence of FMOD after DSS treatment (Fig. 4D). 
A

FMOD +/+
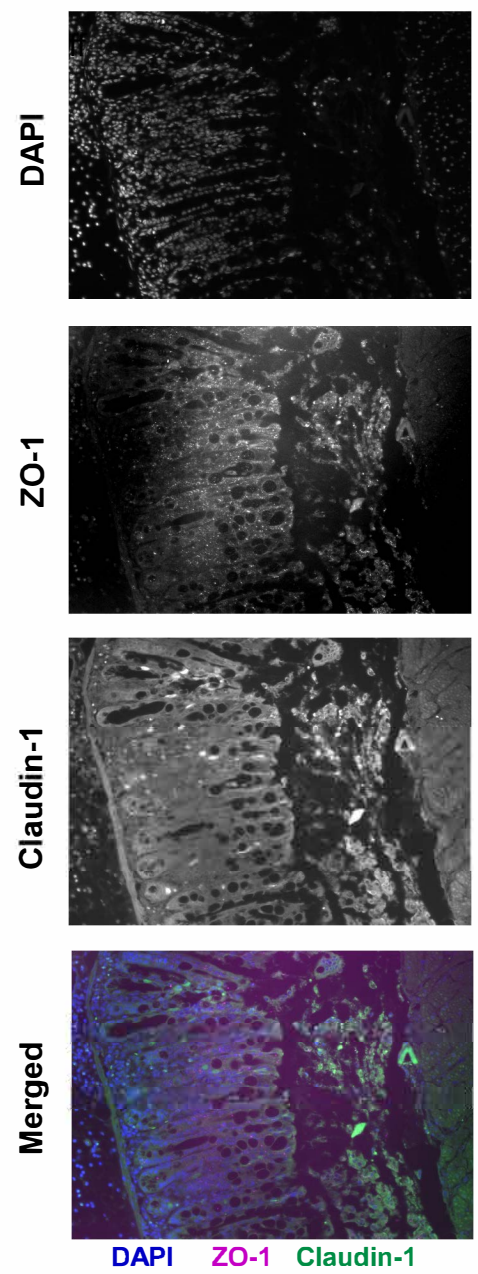

FMOD -/-
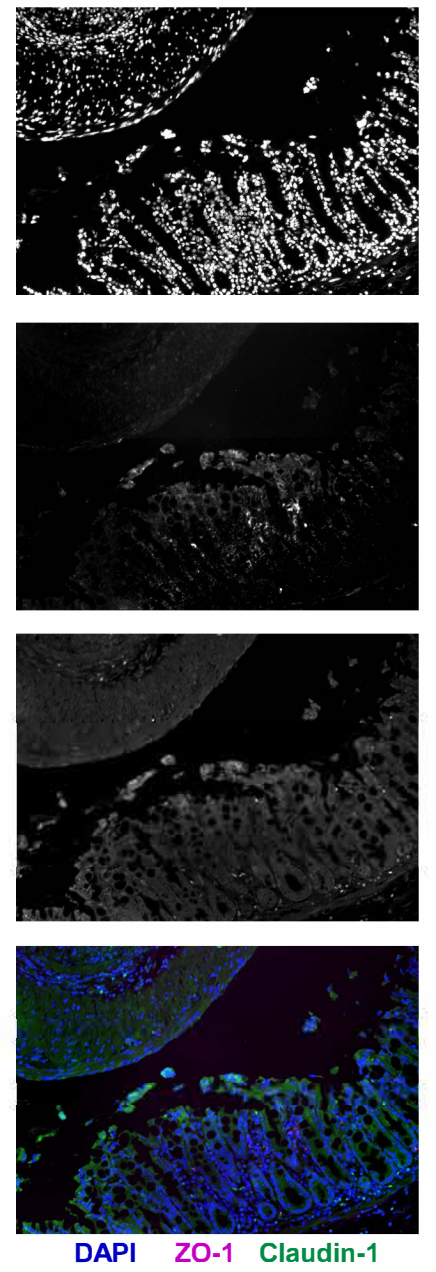

B
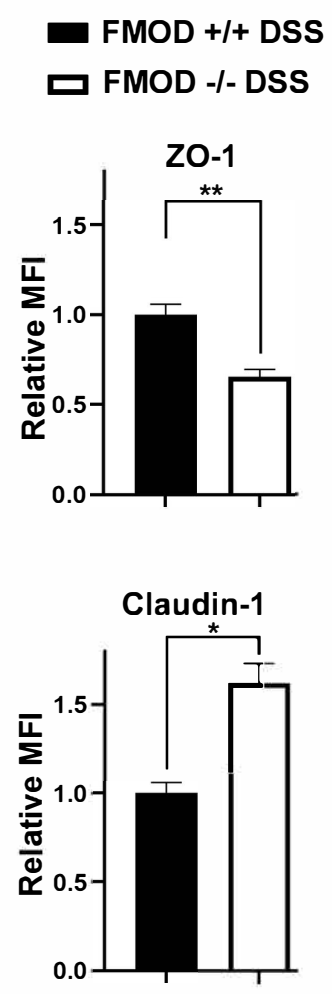

C

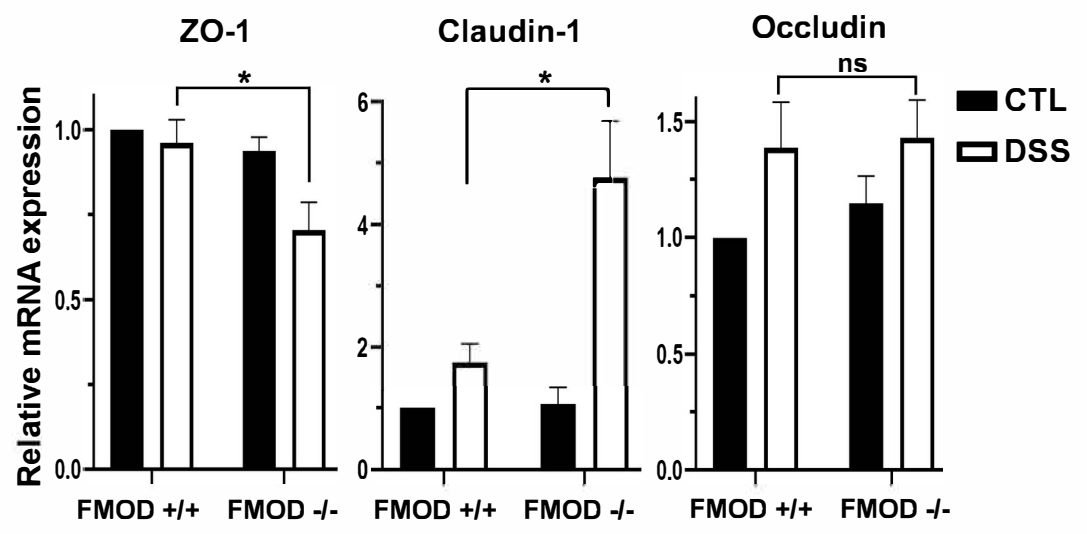

D

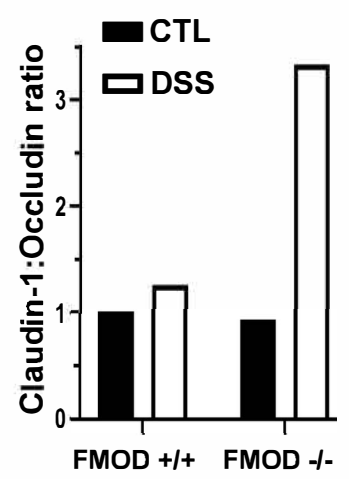




\section{Figure 4. Knockout of FMOD amplifies the changes in the expression of TJ proteins}

following DSS treatment. (A) Paraffin embedded sections of the DSS treated colon Swiss rolls were stained with anti-mouse ZO-1 or claudin-1. Images were captured at x20 magnifications. (B) Graph shows quantification of the mean fluorescence intensity (MFI). (C) The transcriptional expression of TJ proteins ZO-1, claudin-1 and occludin was measured by qRTPCR in the colon of untreated and DSS-treated FMOD+/+ and FMOD-/- mice. Graphs show quantification as fold of mRNA expression levels compared to untreated FMOD $+/+$ mice $(\mathrm{n}=3)$, mean \pm SEM 3 (FMOD+/+) or 4 (FMOD-/-) control and 8 (FMOD+/+) or 10 (FMOD-/-) treated mice. (D) Claudin-1:occludin ratio was calculated using the respective mRNA expression levels. Statistical analysis was performed by unpaired, two-tailed $t$ test with Welch's correction:

$* P<0.05, * * P<0.01$

\section{FMOD alleviates DSS-induced colonic inflammation}

Leukocyte infiltration has been associated with the pathogenesis of human IBD and it also had been implicated in the acute phase of DSS-induced colitis ${ }^{24-26}$. The presence of leukocytes in the colonic tissue was assessed by immunostaining for CD45 on the colon sections of DSS treated mice. Higher expression of CD45 (Fig. 5A) was observed in DSS treated FMOD-/- animals suggesting that inflammation becomes more robust in the absence of FMOD.

Colonic inflammation was further examined by assessing cytokine production in the colonic tissue and the immune response in mesenteric lymph nodes (mLNs), which is known to drain the colonic tissue ${ }^{27}$. First, the production of inflammatory mediators was measured in colon tissue samples by qRT-PCR. Markedly higher mRNA expression of immunomodulatory cytokine interferon $\alpha / \beta$ (IFN $\alpha / \beta$ ) was observed in the DSS-treated FMOD-/- animals, underscoring the development of a potentially more robust inflammatory response (Fig. 5B).

To characterize the recruited and activated immune cell populations in the systemic organs following DSS exposure, mLNs and spleens were harvested and analyzed by flow cytometry. A decrease in the splenic dendritic cell (DC) and T cell populations in both DSS treated groups was detected (data not shown). In the mLNs, significantly more plasmacytoid dendritic cells (pDCs) with increased MHC II (major histocompatibility complex II) maturation markers on their surface were observed in the treated FMOD-/- animals (Fig. 5C). Analysis of T cells revealed significantly higher number of activated T cells in the DSS treated FMOD-/- 
group, while increased mobilization of FoxP3 + T reg cells was observed in the FMOD+/+ DSS treated mice (Fig. 5D), corroborating the presence of a stronger inflammatory response in the background of FMOD KO after DSS administration.

These results suggest that in the absence of FMOD, acute inflammation, represented by activated pDCs and reduced number of T regulatory cells, is significantly increased.

A

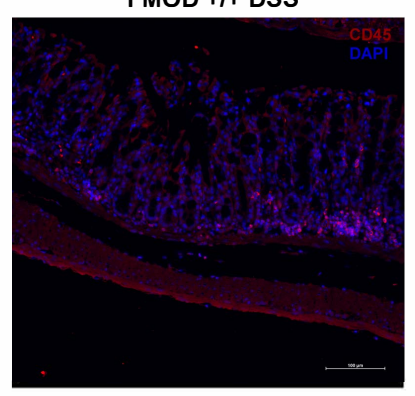

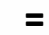

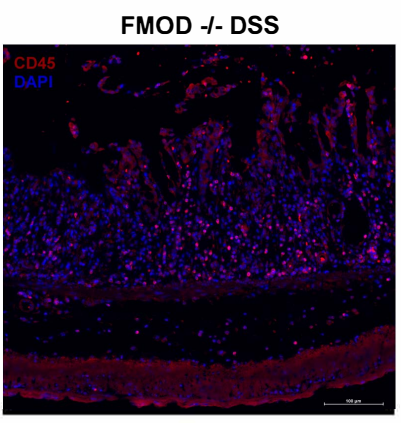

B

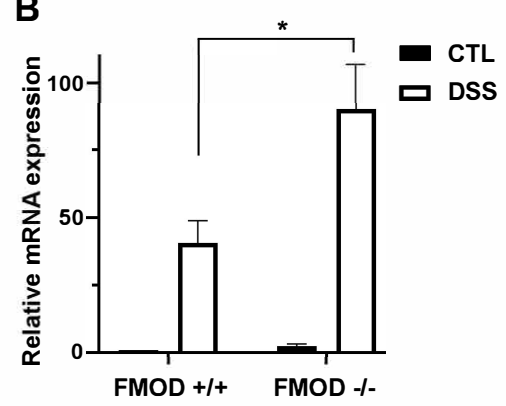

C

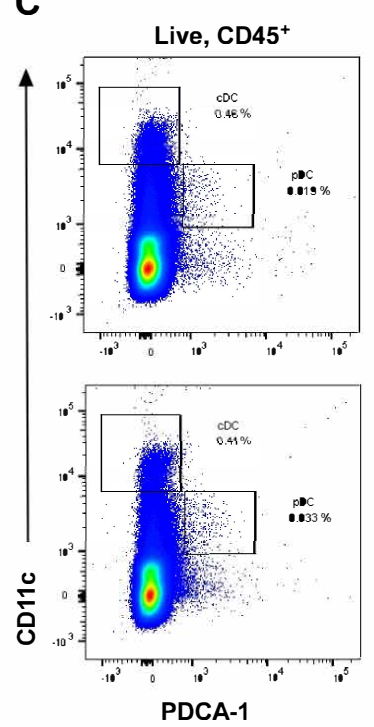

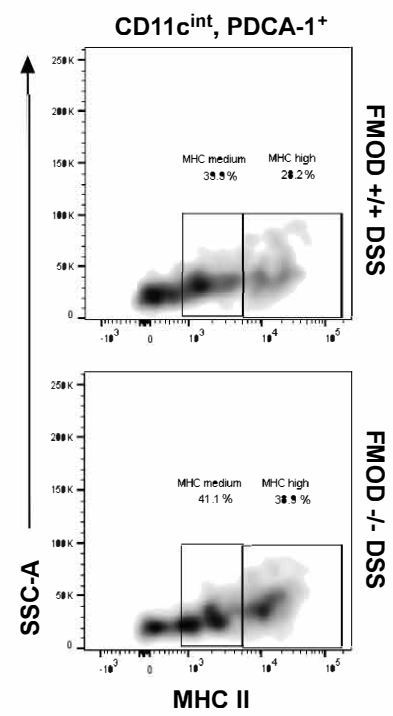

D

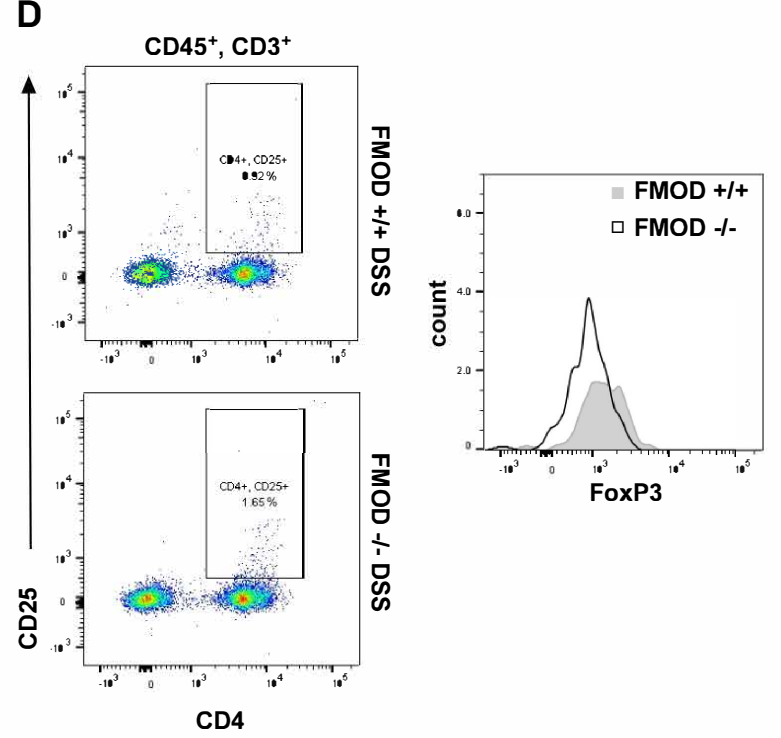

Figure 5. FMOD moderates DSS-induced colonic inflammation. (A) Paraffin embedded sections of the DSS treated colon Swiss rolls were stained with anti-mouse CD45. Images were captured at x20 magnifications. (B) The transcriptional expression of IFN $\alpha / \beta$ was measured by qRT-PCR in the colon. Graph shows quantification as fold of mRNA expression levels compared to untreated FMOD+/+ mice $(n=3)$, mean \pm SEM $3($ FMOD $+/+)$ or 4 (FMOD-/-) control and 8 (FMOD+/+) or 10 (FMOD-/-) treated mice. $* P<0.05$ by unpaired, two-tailed $t$ test with Welch's correction. (C) The mesenteric lymph nodes (mLN) of DSS treated FMOD+/+ $(\mathrm{n}=5)$ and FMOD-/- $(\mathrm{n}=5)$ mice were analyzed by flow cytometry for the frequency (left panel) 
and the maturity (right panel) of pDCs. Data are shown as a dot plot (frequency) or a density

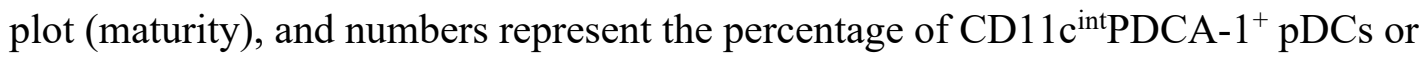

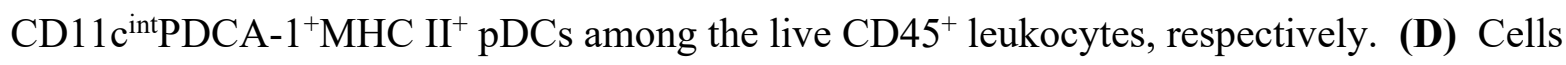
from the mLN of treated FMOD+/+ and FMOD-/- mice were stained for the expression of CD25 and FoxP3 and analyzed by flow cytometry. Dot plots show CD25 and CD4 staining pre-gated on live, $\mathrm{CD} 45^{+} \mathrm{CD}^{+}$cells. The histogram represents FoxP3 staining pre-gated on live, $\mathrm{CD} 45^{+} \mathrm{CD} 3{ }^{+} \mathrm{CD} 4{ }^{+} \mathrm{CD} 25^{+}$cells.

\section{DISCUSSION}

Ulcerative colitis is an idiopathic IBD limited to the colon. It is multifactorial characterized by the dysregulation of the immune system, epithelial barrier defects, and changes in the intestinal microbiota. Based on our earlier findings ${ }^{12}$, we hypothesized that FMOD, a prominent ECM protein, has a role in inflammation and in the maintenance of the epithelial barrier. This study investigates the potential role of FMOD in UC pathogenesis utilizing the widely used DSS-induced acute colitis mouse model.

FMOD, a cytosolic protein with a known secretory sequence, but no transmembrane domain is characterized as a class II member of the small leucin-rich proteoglycan family ${ }^{28,29}$. It was first described in human articular cartilage ${ }^{30}$, and though it is ubiquitously expressed in all tissues among vertebrates, FMOD expression is the most abundant in the extracellular matrix $(E C M)$ of connective tissues, where it has a key role in the organization of collagen fibrils ${ }^{31}$. In recent years, additional tissue specific activities of FMOD such as roles in muscle development ${ }^{32}$, cell reprogramming ${ }^{33}$, angiogenesis, and regulation of TGF $\beta-1$ secretion have been reported 12,34,35. FMOD has also been associated with inflammatory diseases of the joint and antiinflammatory responses in wound healing ${ }^{36}$. To date, the role of FMOD in inflammatory and autoimmune diseases has been modestly explored. Increased expression of FMOD was observed in patients with fibrotic kidney disease ${ }^{37}$, heart failure and atherosclerosis ${ }^{36}$.

Through our experiments, we observed that in both IBD patients and animals with DSSinduced acute colitis, FMOD expression was upregulated during the disease (Fig. 1). This observation may not be too surprising, since colitis is recognized to cause oxidative stress, which is a well-known factor to stimulate the expression of FMOD ${ }^{38}$. Interestingly though, deletion of FMOD in a transgenic acute colitis mouse model did not alleviate disease burden, but rather 
intensified the onset and severity of the clinical colitis symptoms as measured by weight loss, rectal bleeding, and DAI score (Fig. 2). In addition, histopathological examination of full-length colon sections underscored the development of more severe colitis in the absence of FMOD (Fig. 3).

The epithelial barrier physically separates the host immune cells in the intestinal mucosa from the luminal microbiota. Damage to the integrity of the colonic epithelia increases mucosal permeability because of the dysregulation of the TJ proteins ${ }^{2,39}$. Although, epithelial barrier erosion is a key feature of clinical IBD it is still in debate if such dysfunction is a manifestation or a cause of $\mathrm{UC}^{40,41}$. Loss and redistribution of tight junction proteins are commonly reported in acute DSS colitis ${ }^{21}$. In agreement with these findings, marked differences in mRNA and protein expression of TJ proteins ZO-1 and claudin-1 were observed between the DSS treated groups (Fig. 4), showing more robust changes in the absence of FMOD.

Further investigation into the effect of FMOD depletion on colitis revealed the presence of a stronger inflammatory immune response in DSS-treated FMOD-/- mice (Fig. 5). Studies have shown that FMOD plays an important role in inflammatory responses through activating the classical and alternative complement pathways by directly binding to $\mathrm{C} 1 \mathrm{q}$, and $\mathrm{C} 3 \mathrm{~b}^{10}$. The activated complement system contributes to the adaptive and cellular immune responses by interacting with TLRs, antigen-presenting cells, and immune cells such as polymorphonuclear leukocytes, DCs, B cells, as well as T lymphocytes ${ }^{10,42}$. Interestingly, the deposition of C1q can also inhibit molecular processes required for DC differentiation and IFN- $\alpha$ production by pDCs 43-45. Our results align with these previously described findings, because in FMOD+/+ animals the presence of FMOD probably via complement activation induces a more protective immune response as detected by the higher number of Foxp3 $+\mathrm{T}$ reg cells. The absence of FMOD in FMOD-/- animals is associated with increased production of type I IFN, higher numbers of pDCs, and activated T cells indicating a stronger inflammatory response (Fig. 5).

In conclusion, we provide insight into the connection between FMOD and increased susceptibility to colitis by employing genetic and biochemical approaches. In the acute phase of DSS-induced colitis, the presence of FMOD alleviates disease symptoms by maintaining the integrity of the epithelial barrier, reducing pDC activation and inducing regulatory $\mathrm{T}$ cell responses. Future studies are warranted to examine the potential utility of tissue FMOD as a biomarker to determine disease severity of UC. 


\section{ACKNOWLEDGMENT}

The authors thank Professors Rivka Dresner-Pollak, Martin L. Yarmush, MD, PhD and Yuval Tal, MD, PhD for fruitful discussions. This study was supported in part by a grant from NIH (RO1EY024046). 


\section{REFERENCES}

1. Zhou, J., et al. BLT1 in dendritic cells promotes Th1/Th17 differentiation and its deficiency ameliorates TNBS-induced colitis. Cell Mol Immunol 15, 1047-1056 (2018).

2. Ordas, I., Eckmann, L., Talamini, M., Baumgart, D.C. \& Sandborn, W.J. Ulcerative colitis. Lancet 380, 1606-1619 (2012).

3. Zou, J., Liu, C., Jiang, S., Qian, D. \& Duan, J. Cross Talk between Gut Microbiota and Intestinal Mucosal Immunity in the Development of Ulcerative Colitis. Infect Immun 89, e0001421 (2021).

4. Torres, J., Mehandru, S., Colombel, J.F. \& Peyrin-Biroulet, L. Crohn's disease. Lancet 389, 1741-1755 (2017).

5. Bischoff, S.C., et al. Intestinal permeability--a new target for disease prevention and therapy. BMC Gastroenterol 14, 189 (2014).

6. Paris, L., Tonutti, L., Vannini, C. \& Bazzoni, G. Structural organization of the tight junctions. Biochim Biophys Acta 1778, 646-659 (2008).

7. Banchereau, J. \& Steinman, R.M. Dendritic cells and the control of immunity. Nature 392, 245252 (1998).

8. Rescigno, M., Granucci, F., Citterio, S., Foti, M. \& Ricciardi-Castagnoli, P. Coordinated events during bacteria-induced DC maturation. Immunol Today 20, 200-203 (1999).

9. Huang, F.P., et al. A discrete subpopulation of dendritic cells transports apoptotic intestinal epithelial cells to T cell areas of mesenteric lymph nodes. $J$ Exp Med 191, 435-444 (2000).

10. Sjoberg, A., Onnerfjord, P., Morgelin, M., Heinegard, D. \& Blom, A.M. The extracellular matrix and inflammation: fibromodulin activates the classical pathway of complement by directly binding C1q. J Biol Chem 280, 32301-32308 (2005).

11. Merle, N.S., Noe, R., Halbwachs-Mecarelli, L., Fremeaux-Bacchi, V. \& Roumenina, L.T. Complement System Part II: Role in Immunity. Front Immunol 6, 257 (2015).

12. Adini, I., et al. Melanocyte-secreted fibromodulin promotes an angiogenic microenvironment. $J$ Clin Invest 124, 425-436 (2014).

13. Adini, I., et al. Melanocyte pigmentation inversely correlates with MCP-1 production and angiogenesis-inducing potential. FASEB J 29, 662-670 (2015).

14. Kontos, C.D. More than skin deep: connecting melanocyte pigmentation and angiogenic diseases. J Clin Invest 124, 76-79 (2014). 
15. Howe, K.L., Reardon, C., Wang, A., Nazli, A. \& McKay, D.M. Transforming growth factor-beta regulation of epithelial tight junction proteins enhances barrier function and blocks enterohemorrhagic Escherichia coli O157:H7-induced increased permeability. Am J Pathol 167, 1587-1597 (2005).

16. Kel, J.M., Girard-Madoux, M.J., Reizis, B. \& Clausen, B.E. TGF-beta is required to maintain the pool of immature Langerhans cells in the epidermis. J Immunol 185, 3248-3255 (2010).

17. Schultz, H.S., et al. Collagen induces maturation of human monocyte-derived dendritic cells by signaling through osteoclast-associated receptor. J Immunol 194, 3169-3179 (2015).

18. Brand, U., et al. Influence of extracellular matrix proteins on the development of cultured human dendritic cells. Eur J Immunol 28, 1673-1680 (1998).

19. Hall, L.J., et al. Induction and activation of adaptive immune populations during acute and chronic phases of a murine model of experimental colitis. Dig Dis Sci 56, 79-89 (2011).

20. Arimura, K., et al. Crucial role of plasmacytoid dendritic cells in the development of acute colitis through the regulation of intestinal inflammation. Mucosal Immunol 10, 957-970 (2017).

21. Poritz, L.S., et al. Loss of the tight junction protein ZO-1 in dextran sulfate sodium induced colitis. J Surg Res 140, 12-19 (2007).

22. Noth, R., et al. Increased intestinal permeability and tight junction disruption by altered expression and localization of occludin in a murine graft versus host disease model. $B M C$ Gastroenterol 11, 109 (2011).

23. Poritz, L.S., Harris, L.R., 3rd, Kelly, A.A. \& Koltun, W.A. Increase in the tight junction protein claudin-1 in intestinal inflammation. Dig Dis Sci 56, 2802-2809 (2011).

24. te Velde, A.A., et al. Comparative analysis of colonic gene expression of three experimental colitis models mimicking inflammatory bowel disease. Inflamm Bowel Dis 13, 325-330 (2007).

25. Yan, Y., et al. Temporal and spatial analysis of clinical and molecular parameters in dextran sodium sulfate induced colitis. PLoS One 4, e6073 (2009).

26. Arseneau, K.O. \& Cominelli, F. Targeting leukocyte trafficking for the treatment of inflammatory bowel disease. Clin Pharmacol Ther 97, 22-28 (2015).

27. Houston, S.A., et al. The lymph nodes draining the small intestine and colon are anatomically separate and immunologically distinct. Mucosal Immunol 9, 468-478 (2016). 
28. Mikaelsson, E., et al. Fibromodulin, an extracellular matrix protein: characterization of its unique gene and protein expression in B-cell chronic lymphocytic leukemia and mantle cell lymphoma. Blood 105, 4828-4835 (2005).

29. Iozzo, R.V. \& Schaefer, L. Proteoglycan form and function: A comprehensive nomenclature of proteoglycans. Matrix Biol 42, 11-55 (2015).

30. Heinegard, D., et al. Two novel matrix proteins isolated from articular cartilage show wide distributions among connective tissues. J Biol Chem 261, 13866-13872 (1986).

31. Lee, Y.H. \& Schiemann, W.P. Fibromodulin suppresses nuclear factor-kappaB activity by inducing the delayed degradation of IKBA via a JNK-dependent pathway coupled to fibroblast apoptosis. J Biol Chem 286, 6414-6422 (2011).

32. Lee, E.J., et al. Fibromodulin: a master regulator of myostatin controlling progression of satellite cells through a myogenic program. FASEB J 30, 2708-2719 (2016).

33. Zheng, Z., et al. Reprogramming of human fibroblasts into multipotent cells with a single ECM proteoglycan, fibromodulin. Biomaterials 33, 5821-5831 (2012).

34. Zheng, Z., et al. Fibromodulin Enhances Angiogenesis during Cutaneous Wound Healing. Plast Reconstr Surg Glob Open 2, e275 (2014).

35. Jan, A.T., Lee, E.J. \& Choi, I. Fibromodulin: A regulatory molecule maintaining cellular architecture for normal cellular function. Int J Biochem Cell Biol 80, 66-70 (2016).

36. Zeng-Brouwers, J., Pandey, S., Trebicka, J., Wygrecka, M. \& Schaefer, L. Communications via the Small Leucine-rich Proteoglycans: Molecular Specificity in Inflammation and Autoimmune Diseases. J Histochem Cytochem 68, 887-906 (2020).

37. Schaefer, L., et al. Small proteoglycans of normal adult human kidney: distinct expression patterns of decorin, biglycan, fibromodulin, and lumican. Kidney Int 58, 1557-1568 (2000).

38. Mormone, E., Lu, Y., Ge, X., Fiel, M.I. \& Nieto, N. Fibromodulin, an oxidative stress-sensitive proteoglycan, regulates the fibrogenic response to liver injury in mice. Gastroenterology $\mathbf{1 4 2}$, 612-621 e615 (2012).

39. Perse, M. \& Cerar, A. Dextran sodium sulphate colitis mouse model: traps and tricks. J Biomed Biotechnol 2012, 718617 (2012).

40. Martini, E., Krug, S.M., Siegmund, B., Neurath, M.F. \& Becker, C. Mend Your Fences: The Epithelial Barrier and its Relationship With Mucosal Immunity in Inflammatory Bowel Disease. Cell Mol Gastroenterol Hepatol 4, 33-46 (2017). 
41. Lee, J.Y., et al. Molecular Pathophysiology of Epithelial Barrier Dysfunction in Inflammatory Bowel Diseases. Proteomes 6(2018).

42. Heeger, P.S. \& Kemper, C. Novel roles of complement in T effector cell regulation. Immunobiology 217, 216-224 (2012).

43. Son, M., Santiago-Schwarz, F., Al-Abed, Y. \& Diamond, B. C1q limits dendritic cell differentiation and activation by engaging LAIR-1. Proc Natl Acad Sci U S A 109, E3160-3167 (2012).

44. Lood, C., et al. C1q inhibits immune complex-induced interferon-alpha production in plasmacytoid dendritic cells: a novel link between C1q deficiency and systemic lupus erythematosus pathogenesis. Arthritis Rheum 60, 3081-3090 (2009).

45. Bencze, D., Fekete, T. \& Pazmandi, K. Type I Interferon Production of Plasmacytoid Dendritic Cells under Control. Int J Mol Sci 22(2021). 
bioRxiv preprint doi: https://doi.org/10.1101/2022.01.12.475640; this version posted January 12, 2022. The copyright holder for this preprint (which was not certified by peer review) is the author/funder. All rights reserved. No reuse allowed without permission. 